\title{
The IERS Conventions (2010): reference systems and new models
}

\author{
B. Luzum ${ }^{1}$ and G. Petit ${ }^{2} \dagger$ \\ ${ }^{1}$ Earth Orientation Department, U.S. Naval Observatory, Washington, DC 20392, USA \\ ${ }^{2}$ Bureau International des Poids et Mesures, Sèvres, France
}

\begin{abstract}
The IERS Conventions (2010) provides the international standard for models for use in the generation of celestial reference systems (CRS), terrestrial reference systems (TRS), and the Earth orientation parameters (EOPs) that relate the associated frames. Significant improvements over the previous IERS Conventions (2003) are outlined, and an overview of the latest adopted models and standards is shown. Finally, future plans for the Conventions are provided.
\end{abstract}

Keywords. standards, reference systems, time, Earth orientation, astrometry

\section{Introduction}

The IERS Conventions (2010) (Petit \& Luzum 2010) is a set of constants, models, and algorithms, assembled and verified by subject matter experts, to be used in the analysis of Earth orientation and reference systems data. The Conventions strives to be internally consistent as well as consistent with IERS products including the ITRF and ICRF as well as the EOPs that link the two reference frames. Whenever possible, the Conventions are consistent with international standards. Because of the efforts to maintain internal and external consistency, implementation of the IERS Conventions in reduction and analysis software helps to minimize systematic errors in the resulting EOP and reference frame data.

\section{Content}

The IERS Conventions (2010) contains the latest adopted models and standards. Topics included are numerical standards, reference systems, transformations between systems, geopotential, displacement of reference points, tidal variations, atmospheric propagation delays, and relativistic models. The recommended models are reviewed continually, and suggested improvements are evaluated by an Advisory Board. Based on their recommendation, modifications are made to the content of the IERS Conventions Updates and ultimately result in a new registered edition.

\section{Improvements in IERS Conventions (2010)}

Several improvements were made to the IERS Conventions (2010). The most significant changes to the Conventions came with the adoption of the ITRF2008 (Altamimi et al. 2011), ICRF2 (Fey et al. 2009), and the IAU2000/2006 precession-nutation model (Mathews et al. 2002, Capitaine et al. 2003). Additional improvements in modeling were

$\dagger$ E-mail: brian.luzum@usno.navy.mil or gpetit@bipm.org 
achieved through the adoption of a new conventional geopotential model and new tropospheric propagation model/zenith mapping function.

To reduce confusion, classification of models were introduced and the criteria for choosing models was stated explcitly. Tables with the magnitude of the modeled effects were provided and better consistency throughout the document was enforced. Significant efforts were made to improve the standardization of software including the creation of a new software template, improved documentation, improved robustness of the code, and the inclusion of test cases. In assembling the software, efforts were made to cooperate closely with the IAU Standards of Fundamental Astronomy (SOFA) software.

\section{Future changes}

To keep the IERS Conventions current, additional changes are being considered. Several models can be improved, and efforts are underway to incorporate, for example, an improved global pressure and temperature model, diurnal and semidiurnal EOP variation model, conventional mean pole, ionospheric correction to ray bending, and a geocenter model. The sections on displacement of reference points and on ranging techniques will be expanded. Also, non-tidal loading and a SINEX format for modeling will be investigated.

\section{Acknowledgements}

We would like to acknowledge the extensive work of the Advisory Board, led by Jim Ray, and all of the contributors who have helped make the IERS Conventions (2010) the high-quality document that it is.

\section{References}

Altamimi, Z., Collilieux, X., \& Métivier, L. 2011, J. Geod. 85, 457

Capitaine, N., Wallace, P. T., \& Chapront, J. 2003, Astron. Astrophys. 412, 567

Fey, A. L., Gordon, D., \& Jacobs, C. S. 2009, IERS Technical Note 35, 204 pp

Mathews, P. M., Herring, T. A., \& Buffett, B. A. 2002, J. Geophys. Res. 107, doi: 10.1029/2001JB000390

Petit, G. \& Luzum, B. 2010, IERS Technical Note 36, 179 pp 